\title{
Psychogenetics of post-traumatic stress disorder: a short review
}

\author{
This article was published in the following Dove Press journal: \\ The Application of Clinical Genetics \\ 15 November 2010 \\ Number of times this article has been viewed
}

\author{
Ahmed Rady \\ Adel Elsheshai \\ Osama Elkholy \\ Heba Abou el Wafa \\ Department of Psychiatry, Alexandria \\ University, Alexandria, Egypt
}

\begin{abstract}
Post-traumatic stress disorder is a commonly overlooked psychiatric disorder due to the heterogeneity of symptoms that may simulate many other psychiatric disorders. Such heterogeneity of manifestations may be explained by the multifaceted nature of the different neurotransmitters, endocrinologic axis, and their genetic basis, that are implicated in the etiology. Although this disorder has been studied from many different perspectives, its etiology is still enigmatic. This minireview demonstrates, in brief, that different susceptibility genes are associated with post traumatic stress disorder.
\end{abstract}

Keywords: trauma, post traumatic stress disorder, psychogenetic, stress response, neurobiology

\section{Introduction}

Although the lifetime prevalence of exposure to traumatic events is thought to be between $40 \%$ and $90 \%$ in the general population, the overall lifetime prevalence of post-traumatic stress disorder (PTSD) is estimated at 7\%-12\%. This means that exposure to a traumatic event does not entirely explain the etiology of the disorder. The suspicion is that individuals with an existing genetic vulnerability have a higher risk of developing PTSD once they experience a trauma. Two sources of evidence support a genetic component, ie, transgenerational research and epidemiologic studies in twins. Transgenerational studies have reported that PTSD is more likely to occur in certain families. Twin studies have found that monozygotic twins are more concordant for developing PTSD after trauma exposure than dizygotic twins. ${ }^{1-4}$

Although evidence exists for a genetic vulnerability to PTSD, the vulnerability develops in interaction with environmental factors, such as stressful life events or a mother's prepartum or even prefertilization hormonal status. The etiology of PTSD is complex and multifactorial, and like most other mental disorders, the heritable part of PTSD can be viewed as polygenetic. This means that different genes are assumed either to interact or to play an additional role in the ultimate onset of the disorder. ${ }^{5}$

The complexity of psychiatric disorders like PTSD makes it difficult to find specific genes that contribute substantially to the disorder. For this reason, genetic research often trains its focus on endophenotypes, ie, more elementary underlying traits or facets of clinical phenomena, whereby the number of genes required to produce variations in these traits may be fewer than those involved in producing a psychiatric diagnostic entity. Such basic traits may consist of neurophysiologic, biochemical, endocrinologic, neuroanatomic, cognitive, and neuropsychologic measures. Authors have argued that genes associated with certain endophenotypes may be implicated in development of PTSD. These endophenotypes for PTSD include the dysregulation 
of the hypothalamus-pituitary-adrenal (HPA) axis, the physiology of hyperarousal, and the exaggerated acoustic startle response. Other important response measures include autonomic reactivity, such as heart rate variability, and psychologic variables, including memory problems. ${ }^{6,7}$

Linkage studies investigate at random the entire genomes of individuals, using DNA markers to locate chromosomal areas that are continuously passed on together with a particular disorder. Natural genetic variants are sought, and their prevalence is assessed in subjects with a disorder like PTSD against healthy control groups.

Most of the hypotheses on candidate genes implicated in PTSD derive from linkage studies in animals. Candidate genes identified so far are the serotonin transporter gene (5-HTT), dopamine receptor gene (DRD2) and dopamine transporter gene (DAT), glucocorticoid receptor gene (GR), GABA (A) receptor gene, apolipoprotein E (ApoE) gene, brain-derived neurotrophic factor gene (BDNF), and neuropeptide Y gene (NPY). ${ }^{8}$

A next step in molecular genetic research is to investigate in association studies whether a connection exists between a genetic variant (polymorphism) and an endophenotype in a disorder. Studies like these can uncover smaller genetic effects. Therefore, they seem the method best suited for molecular genetic studies in complex disorders, including PTSD. The presence of genetic polymorphisms in candidate genes in a group of individuals with a particular disorder is compared with a group of healthy individuals. Single nucleotide polymorphisms (SNPs) are small changes in DNA that have no visible effects but do affect vulnerability. A distinction is made between functional and nonfunctional SNPs. ${ }^{9}$

Functional SNPs have effects on gene expression and/or protein function. No such effects are known from nonfunctional SNPs, but they can still be of use for association studies. Although they probably do not cause the disorder, they lie on a chromosome near the "pathogenic" mutation. Candidate genes investigated in association studies should preferably have already been identified in linkage analyses, or should be part of a well defined prior hypothesis. Because linkage analysis is difficult to perform in PTSD, candidate genes not identified in prior human linkage analyses are often selected for association studies on the basis of hypotheses about their putative functional relationship with PTSD (or after identification in animal studies). Should an association then be found, it merely shows that the polymorphism in question is somehow connected to a particular endophenotype of PTSD, but it does not demonstrate a causal relationship. ${ }^{10}$

Other as yet undiscovered factors, such as a link between that polymorphism and another polymorphism in the same gene or a nearby gene, could explain the association. The genetic research conducted at present in relation to PTSD involves these types of association studies on candidate genes preselected without prior human linkage analysis. ${ }^{11}$

A relatively new method of genetic research is to study expression of genes in specific tissues by microarray analysis using an RNA or cDNA chip. This was recently done for the first time in PTSD. Segman et al used oligonucleotide microarrays to measure peripheral blood mononuclear cell gene expression in trauma survivors directly after they presented to a casualty department and 4 months later. ${ }^{12}$ The results showed an overall reduction in expression of transcription activators of peripheral blood mononuclear cells in psychologically distressed victims. This demonstrates the possibility of stress-induced reduction of gene expression. ${ }^{12,13}$

\section{Genes involved in the serotonin system}

Evidence exists that the genes regulating the serotonin system play a role in susceptibility to PTSD or to depression, in response to various types of stressors over the life course. ${ }^{13}$

The serotonin transporter gene has been identified in relation to the serotonin system and PTSD. A great deal of research has already been done on genes involved in the serotonin system, and which thereby affect serotonergic transmission. The serotonin transporter, 5-HTT (also called SERT), is the best studied biologic substrate of depression. Abnormal serotonergic activity may mediate susceptibility to affective disorders, and a relationship has also been found with stress reactions. ${ }^{14}$

Initially, considerable experimental research was done in animals which supported the hypothesis of a "gene-by-environment" interaction, and showed that variation in the 5-HTT transporter gene was linked to altered serotonergic function following stressful early life events. For example, serotonin played a part in HPA axis alterations in animals exposed to early life stress. ${ }^{15}$

Although the role of serotonergic transmission in the pathophysiology of PTSD is still unclear, serotonin is thought to be involved in the onset of the PTSD symptoms relating to mood, arousal, and sleep. Allelic variation in human 5-HTT expression is caused by functional gene promoter polymorphisms with two predominant variant alleles, which are likewise associated with various anxiety responses to stressful events. ${ }^{16}$

In vitro studies have shown basal 5-HTT activity in carriers of the 5-HTTPR long (L) allele to be twice as high as in carriers of the short $(\mathrm{S})$ allele, indicating that 5-HTT 
gene transcription may be modulated by these variants. An association study in individuals with PTSD versus a healthy control group has found only marginal differences in genotypes between the two groups, except that the frequency of the SS genotype was significantly higher in the PTSD group. A limitation was that the controls had never experienced a trauma, so it was unknown whether some might have developed PTSD on trauma exposure. ${ }^{17}$

A review of neuroimaging studies found that people with an SS or SL allele showed greater amygdala activation in response to fearful stimuli than those with the LL allele. In another study, neurochemical processes were associated with genetic variations in serotonergic neurotransmission relevant to anxiety. Significantly lower hippocampal N-acetylaspartate concentrations were found in SL carriers than in individuals with an LL genotype, and $\mathrm{N}$-acetylaspartate concentrations correlated negatively with anxiety traits on the Spielberger State-Trait Anxiety Inventory. ${ }^{18,19}$

\section{Genes involved in the dopamine system}

There are two important PTSD candidate genes that directly affect the dopamine system, ie, DRD2 and DAT. The DRD2 minor (A1) allele, DRD2 A1, has already been linked to attention deficit hyperactivity disorder, Tourette's syndrome, conduct disorder, and substance abuse. ${ }^{20}$ This prompted suppositions that this gene may be involved in the stress response in humans. ${ }^{20}$ The first study of the role of DRD2 in PTSD was published by Comings et al. ${ }^{21}$ Although it reported a significant association between the presence of the DRD2 A1 allele and PTSD, that was not confirmed in a later study. ${ }^{21,22}$

There has been only one study of the DAT gene in relation to PTSD. In a large group of twin pairs who had been in the Vietnam war, researchers sought to establish a link between PTSD and the DAT SLC6A3 30-variable number tandem repeat, using a trauma-exposed control group without PTSD. Evidence was found that genetically determined changes had occurred in dopaminergic reactivity among the PTSD subjects. ${ }^{23}$

\section{Genes involved in the glucocorticoid system}

An earlier animal study found that two hippocampal GR receptors played a key role in regulating the HPA axis and cortisol levels. These are the mineralocorticoid (MR, or Type 1) receptor encoded on chromosome 5 and the GR (or Type 2) receptor encoded on chromosome 4. Because MRs have high affinity to cortisol (10 times greater than that of GRs), it is mainly MRs that are occupied in the absence of stress (ie, basal corticosteroid levels). When stress arrives, cortisol levels increase sharply and the GRs are also occupied. Aldosterone plays a part here, and via MR can affect the expression of GR mRNA. The balance between the effects of these two corticosteroid receptor types is critical to stress response and behavioral adaptation thereafter. Both MR- and GR-mediated effects of information processing facilitate behavioral adaptation and thereby stimulate higher brain centers to exert inhibitory control on HPA axis activity. ${ }^{24-26}$

Individual differences in the number, affinity, and efficiency of the signaling cascades activated by these receptors have direct effects on cortisol levels and biologic activity. Corticosteroid receptors function as transcription factors. Most evidence in PTSD and relevant animal models points towards an involvement of altered GR rather than MR function. Much research has already been done on the GR gene and on sensitivity to corticosteroids, and most studies have reported positive associations. Both the N363S and the BclI polymorphisms of this gene have been linked to GR hypersensitivity. ${ }^{27-29}$

Bachmann et al went on to test whether variations in the GR gene showed links to PTSD. ${ }^{30}$ In a group of PTSD and trauma-exposed non-PTSD Vietnam war veterans, Bachmann et al screened for polymorphisms, and assessed GR sensitivity using the low-dose dexamethasone suppression test and the dermal vasoconstrictor assay. The researchers concluded that the N363S and BclI GG genotypes were no more common in PTSD subjects than in the control subjects or in the general population. However, they did find a significant association between the BclI GG genotype and low basal cortisol levels in PTSD. Subjects with PTSD and the GG genotype tended to be more responsive to the dermal vasoconstrictor assay, and their dermal vasoconstrictor assay response correlated with higher scores on the clinician-administered PTSD scale. However, there was insufficient evidence that GR polymorphisms actually increase susceptibility to PTSD. ${ }^{30}$

\section{Genes involved in the GABA system}

Studies on the connections between GABA (the GABA Type A receptor alpha 6 subunit gene, GABRA6) and cortisol have reported that homozygotes for the $\mathrm{T}$ allele had generally higher diurnal cortisol levels, but only one study has sought links between GABA and PTSD. It focused on the GABA (A) receptor beta 3 subunit gene (GABRB3). Individuals with PTSD who were heterozygotic for the G1 polymorphism of $G A B R B 3$ were found to have higher total scores than homozygotic individuals on the General Health 
Questionnaire, the four subscales of which pertain to somatic symptoms, anxiety and insomnia, social dysfunction, and depression. Comorbidity was not an exclusion criterion, the number of persons studied was small, and there was no control group, so the results have to be interpreted with care. In summary, little is known about the GABA system in relation to PTSD. ${ }^{31,32}$

\section{Genes involved in the ApoE system}

In humans, ApoE4 has been linked with a number of neuropsychiatric disorders, including Alzheimer's disease, stress, and depression, as well as with smaller hippocampal volumes and with subjective and objective memory impairment. ApoE2 has been linked to lower cortisol levels. Because memory impairment also occurs in PTSD, smaller hippocampal volumes are sometimes found too. Freeman et al assessed what role the ApoE genotype might play in this disorder. It was found that carriership of allele 2, and not of allele 4, was associated with significantly lower scores on the memory test and with more severe re-experiencing of symptoms. ${ }^{33}$ This study was also limited by its small sample size, psychiatric comorbidity, and lack of a control group. Therefore, there is still insufficient evidence concerning the possible role of the ApoE genotype in relation to PTSD. ${ }^{34,35}$

\section{Genes involved in the brain-derived neurotrophic factor system}

BDNF, a member of the neurotrophin family, promotes neuronal survival and regulates the proliferation and differentiation of nerve cells in the peripheral and central nervous systems. It has important regulatory effects on the serotonergic, glutamatergic, and dopaminergic neurotransmitter systems. $^{36}$

BDNF is also involved in long-term hippocampal potentiation, which is related to learning and memory. There is strong evidence that BDNF may contribute to the pathogenesis of several neuropsychiatric disorders, and it is also believed to be involved in PTSD. Data from an animal study suggest that psychologic stress, as well as unconditioned physical stress, can decrease hippocampal BDNF mRNA, which could be relevant to the pathogenesis of stress-related disorders, including depression and PTSD. ${ }^{36}$

Zhang et al observed the association of gene variants of the BDNF gene and several neuropsychiatric phenotypes. ${ }^{36}$ They compared 69 subjects with PTSD with a healthy control group. The SNPs for G-712A, C270T, and Val66Met were genotyped. There was only an association between the newly described SNP G-712A and substance dependence, but no association of the SNPs with PTSD. Given the low heterozygosity or the low information content of C270T and G-712A, these two polymorphisms appear to require larger numbers of cases to ensure adequate statistical power. In addition, although the overall study sample was large, the sample for PTSD was much smaller and this limited the power to detect significant associations. In the Korean population, Lee et al analysed the genotype and allele frequencies of the BDNF gene Val66Met polymorphism in 106 PTSD patients and 161 unrelated healthy controls using a case-control design. ${ }^{37}$ The genotype and allele frequencies for the BDNF gene polymorphism did not differ between the two groups. ${ }^{37,38}$

\section{Genes involved in the neuropeptide $Y$ system}

NPY is a 36-amino acid peptide neurotransmitter. Animal studies have suggested that NPY is involved in the regulation of appetite, reward, anxiety, and energy balance. NPY is present in extensive neuronal systems of the brain, and is present in high concentrations in cell bodies and terminals in the amygdala. Morgan et al found in two different studies in soldiers without a control group, that acute stress elicits NPY release, and that this release is positively associated with cortisol and norepinephrine release. ${ }^{39}$ The finding that greater levels of NPY release are associated with less psychologic distress suggests that NPY has anxiolytic activity in humans. ${ }^{39-41}$

Individuals with the Pro7/Leu7 genotype have higher maximal increases in plasma concentrations of NPY in response to maximal physiologic stress as compared with Leu7/Leu7 individuals. Lappalainen et al tested whether the Leu 7pro allele is associated with alcohol-dependent subjects in a population study compared with healthy controls. ${ }^{40}$ Population stratification potential and diagnostic specificity were studied by genotyping individuals from additional populations and psychiatric diagnostic classes, such as PTSD. There were 77 PTSD Vietnam-era combat veterans. The main outcome measure was the difference in Leu7Pro allele frequencies between alcohol-dependent subjects and controls. There was no association with PTSD. Until now, there is no evidence for the possible role of gene variants of the NPY gene in relation to PTSD. ${ }^{40-42}$

\section{Genes modulating the hypothalamus-pituitary-adrenal axis}

The HPA axis plays an important role in the stress response. It involves corticotrophin releasing hormone that stimulates, 
in turn, release of adrenocorticotrophic hormone by the pituitary gland which stimulates cortisol secretion by the suprarenal gland. The HPA axis is a complicated axis in terms of the diverse feedback mechanisms involved in its regulation, as well as other GR-regulating genes.

Some authors have questioned dysfunction of the GRR2 gene as a potential cause of dysregulation in HPA axis feedback mechanisms. PTSD patients show baseline hypocortisolemia, and it has been suggested that this hypocortisolemia may reflect a defect in the normal stress-related response. Fear conditioning models have been expanded to incorporate altered post-trauma cortisol response. Low peritrauma cortisol levels may fail to contain the noradrenergic stress response, leading to consequent prolonged increases in levels of norepinephrine in the brain. Research has also implicated glucocorticoids in modulation of extinction of fear memories. Nonetheless, the only published investigation of glucocorticoid genes in PTSD reported no association between two glucocorticoid receptor polymorphisms (N363S and BclI) and PTSD. ${ }^{43-45}$

One key regulating gene of this system is FKPB5. A recent study revealed that polymorphisms in FKBP 5 moderate PTSD on exposure to childhood sexual abuse. Further, dexamethasone suppression test data also demonstrated a GxE interaction between FKBP5 polymorphisms and PTSD status, suggesting that these polymorphisms have functional consequences on glucocorticoid response system sensitivity. Polymorphisms in FKBP5 were also found to be associated with peritraumatic dissociation, which predicts the development of PTSD. Thus, variation in the GR genes (GCCR, GCR2) and glucocorticoid-regulating genes (eg, FKBP5) may be related to increased risk of PTSD. ${ }^{45,46}$

\section{Genetic variation and response to psychotherapy}

An interesting and innovative study was carried out by an Australian team to assess whether a polymorphism of serotonin transporter gene (SLC6A4) may potentially affect response to cognitive behavioral psychotherapy. There are two variations in the region of SLC6A4 that promote the release and uptake of serotonin, an $\mathrm{S}$ allele and a $\mathrm{L}$ allele. The S allele has been linked to increased risk of PTSD in the presence of low social support. Individuals with PTSD who have this polymorphism also show heightened reactivity in the amygdala, involved in fear processing when the individual is exposed to traumatic stimuli. They assessed 45 patients with PTSD over eight weeks, with cognitive behavioral psychotherapy sessions on a weekly basis.
Although the sample size was small and further large-scale studies are needed to replicate this finding, the results were really interesting. The $\mathrm{S}$ and $\mathrm{L}$ allele groups did not differ according to analyses that examined PTSD at pretreatment, post-treatment, and at six-month follow-up, but specific comparisons showed that the $\mathrm{S}$ allele group had greater symptom severity at follow-up, and was more likely than the L allele group to still have PTSD (48\% versus $15 \%$, respectively). ${ }^{47,48}$ To our knowledge, this is the first research dealing with genetic variability in psychotherapy response rate, and may open new perspectives in optimizing PTSD patient management based on genetic analysis.

\section{Conclusion}

PTSD is polymorphic in terms of its symptomatology, etiology, and treatment response. Different candidate susceptibility genes may not only contribute to better understanding of the syndrome but also aid in its future management.

\section{Disclosure}

The authors report no conflicts of interest in this work.

\section{References}

1. Breslau N. The epidemiology of posttraumatic stress disorder: What is the extent of the problem? J Clin Psychiatry. 2001;62:16-22.

2. Olff M, de Vries GJ. Prevalence of trauma and PTSD in The Netherlands. Paper presented at 21 st annual meeting of the International Society for Traumatic Stress Studies. Toronto, Canada, 2005 Nov 2-5.

3. Koenen KC, Lyons MJ, Goldberg J, et al. A high risk twin study of combat-related PTSD comorbidity. Twin Res. 2003;6:218-226.

4. Stein MB, Jang KL, Taylor S, et al. Genetic and environmental influences on trauma exposure and posttraumatic stress disorder symptoms: A twin study. Am J Psychiatry. 2002;159:1675-1681.

5. de Kloet ER, Sibug RM, Helmerhorst FM, et al. Stress, genes and the mechanism of programming the brain for later life. Neurosci Biobehav Rev. 2005;29:271-281.

6. Gottesman II, Gould TD. The endophenotype concept in psychiatry: Etymology and strategic intentions. Am J Psychiatry. 2003;160: 636-645.

7. Segman RH, Shalev AY. Genetics of posttraumatic stress disorder. CNS Spectroscopy. 2003;8:693-698.

8. Gass P, Reichardt HM, Strekalova T, et al. Mice with targeted mutations of glucocorticoid and mineralocorticoid receptors: Models for depression and anxiety? Physiol Behav. 2001;73:811-825.

9. Sullivan PF, Neale MC, Silverman MA, et al. An association study of DRD5 with smoking initiation and progression to nicotine dependence. Am J Med Genet. 2001;105:259-265.

10. van Rossum EF, Russcher H, Lamberts SW. Genetic polymorphisms and multifactorial diseases: Facts and fallacies revealed by the glucocorticoid receptor gene. Trends Endocrinol Metab. 2005;16:445-450.

11. Figee M, van Amelsvoort T, de Haan L, et al. Genetisch onderzoek bij schizofrenie. Tijdschrift voor Psychiatrie. 2004;46:15-25.

12. Segman RH, Shefi N, Goltser-Dubner T, et al. Peripheral blood mononuclear cell gene expression profiles identify emergent post-traumatic stress disorder among trauma survivors. Mol Psychiatry. 2005;10 $500-513$.

13. Broekman BFP, Olff M, Boer F. The genetic background to PTSD Neurosci Biobehav Rev. 2007;31:348-362. 
14. Stahl SM. Is psychopharmacologic 'inoculation' effective in preventing posttraumatic stress disorder? J Clin Psychiatry. 2005;66:5-6.

15. Caspi A, Sugden K, Moffitt TE, et al. Influence of life stress on depression: Moderation by a polymorphism in the 5-HTT gene. Science. 2003;301:386-389.

16. Bennett AJ, Lesch KP, Heils A, et al. Early experience and serotonin transporter gene variation interact to influence primate CNS function. Mol Psychiatry. 2002;7:118-122.

17. Glatz K, Mossner R, Heils A, et al. Glucocorticoid regulated human serotonin transporter (5-HTT) expression is modulated by the 5-HTT gene-promoter-linked polymorphic region. $J$ Neurochem. 2003;86: 1072-1078

18. Lee HJ, Lee MS, Kang RH, et al. Influence of the serotonin transporter promoter gene polymorphism on susceptibility to posttraumatic stress disorder. Depress Anxiety. 2005;21:135-139.

19. Gallinat J, Strohle A, Lang UE, et al. Association of human hippocampal neurochemistry, serotonin transporter genetic variation, and anxiety. Neuroimage. 2005;26:123-131.

20. Noble EP. The DRD2 gene in psychiatric and neurological disorders and its phenotypes. Pharmacogenomics. 2000;1:309-333.

21. Comings DE, Muhleman D, Gysin R. Dopamine D2 receptor (DRD2) gene and susceptibility to posttraumatic stress disorder: A study and replication. Biol Psychiatry. 1996;40:368-372.

22. Gelernter J, Southwick S, Goodson S, et al. No association between D2 dopamine receptor (DRD2) 'A' system alleles, or DRD2 haplotypes, and posttraumatic stress disorder. Biol Psychiatry. 1999;45:620-625.

23. Segman RH, Shalev AY. Genetics of posttraumatic stress disorder. CNS Spectroscopy. 2003;8:693-698.

24. Liberzon I, Lopez JF, Flagel SB, et al. Differential regulation of hippocampal glucocorticoid receptors mRNA and fast feedback: Relevance to post traumatic stress disorder. J Neuroendocrinol. 1999;11:11-17.

25. de Kloet ER, Vreugdenhil E, Oitzl AS. Brain corticosteroid receptor balance in health and disease. Endocr Rev. 1998;19:269-301.

26. Gass P, Reichardt HM, Strekalova T, et al. Mice with targeted mutations of glucocorticoid and mineralocorticoid receptors: Models for depression and anxiety? Physiol Behav. 2001;73:811-825.

27. de Kloet ER, Derijk R. Signaling pathways in brain involved in predisposition and pathogenesis of stress-related disease: Genetic and kinetic factors affecting the MR/GR balance. Ann NY Acad Sci. 2004; 1032:14-34

28. Yehuda R, Golier JA, Yang RK, et al. Enhanced sensitivity to glucocorticoids in peripheral mononuclear leucocytes in posttraumatic stress disorder. Biol Psychiatry. 2004;55:1110-1116.

29. van Rossum EF, Koper JW, van den Beld AW, et al. Identification of the BclI polymorphism in the glucocorticoid receptor gene: Association with sensitivity to glucocorticoids in vivo and body mass index. Clin Endocrinol. 2003;59:585-592.

30. Bachmann AW, Sedgley TL, Jackson RV, et al. Glucocorticoid receptor polymorphisms and post-traumatic stress disorder. Psychoneuroendocrinology. 2005;30:297-306.

31. Feusner J, Ritchie T, Lawford B, et al. GABA-A receptor beta 3 subunit gene and psychiatric morbidity in a post-traumatic stress disorder population. Psychiatry Res. 2001;104:109-117.

32. Rosmond R, Bouchard C, Bjorntorp P. Allelic variants in the GABA(A) alpha6 receptor subunit gene (GABRA6) is associated with abdominal obesity and cortisol secretion. Int J Obes Related Metab Disord. 2002;26:938-941

The Application of Clinical Genetics

\section{Publish your work in this journal}

The Application of Clinical Genetics is an international, peer-reviewed open access journal that welcomes laboratory and clinical findings in the field of human genetics. Specific topics include: Population genetics; Functional genetics; Natural history of genetic disease; Management of genetic disease; Mechanisms of genetic disease; Counselling and
33. Freeman T, Roca V, Guggenheim F, et al. Neuropsychiatric associations of apolipoprotein E alleles in subjects with combat-related posttraumatic stress disorder. J Neuropsychiatry Clin Neurosci. 2005;17:541-543.

34. Gallagher-Thompson D, O'Hara R, Simmons A. Apolipoprotein E epsilon 4 allele affects the relationship between stress and depression in caregivers of patients with Alzheimer's disease. J Geriatr Psychiatry Neurol. 2001;14:115-119.

35. Peskind ER, Wilkinson CW, Petrie EC. Increased CSF cortisol in AD is a function of APOE genotype. Neurology. 2001;56:1094-1108.

36. Zhang H, Ozbay F, Lappalainen J, et al. Brain derived neurotrophic factor (BDNF) gene variants and Alzheimer's disease, affective disorders, posttraumatic stress disorder, schizophrenia, and substance dependence. Am J Med Genet B Neuropsychiatr Genet. 2006;141:387-393.

37. Lee HJ, Kang RH, Lim SW, et al. No association between the brain-derived neurotrophic factor gene Val66Met polymorphism and post-traumatic stress disorder. Stress Health. 2006;22:115-119.

38. Rasmusson AM, Shi L, Duman R. Downregulation of BDNF mRNA in the hippocampal dentate gyrus after re-exposure to cues previously associated with footshock. Neuropsychopharmacology. 2002;27: 133-142.

39. Morgan III CA, Wang S, Southwick SM, et al. Plasma neuropeptide-Y concentrations in humans exposed to military survival training. Biol Psychiatry. 2000;47:902-909.

40. Lappalainen J, Kranzler HR, Malison R, et al. A functional neuropeptide Y Leu7Pro polymorphism associated with alcohol dependence in a large population sample from the United States. Arch Gen Psychiatry. 2002;59:825-831.

41. Morgan III CA, Rasmusson AM, Wang S, et al. Neuropeptide-Y, cortisol, and subjective distress in humans exposed to acute stress: Replication and extension of previous report. Biol Psychiatry. 2002;52:136-142.

42. Kallio J, Pesonen U, Kaipio K, et al. Altered intracellular processing and release of neuropeptide $Y$ due to Leucine 7 to Proline 7 polymorphism in the signal peptide of preproneuropeptide $\mathrm{Y}$ in humans. FASEB J. 2001;15:1242-1244.

43. Yang Y, Chao P, Lu K. Systemic and intra-amygdala administration of glucocorticoid agonist and antagonist modulate extinction of conditioned fear. Neuropsychopharmacology. 2006;31:912-924.

44. Bachmann AW, Sedgley TL, Jackson RV, et al. Glucocorticoid receptor polymorphism and post-traumatic stress disorder. Psychoneuroendocrinology. 2005;30:297-306.

45. Amstadter AB, Nugent NR, Koenen KC. Genetics of PTSD: Fear conditioning as a model for future. Psychiatr Ann. 2009;39:358-367.

46. Binder EB, Bradley RG, Liu W, et al. Association of FKBP5 polymorphisms and childhood abuse with risk of posttraumatic stress disorder symptoms in adults. JAMA. 2008;299:1291-1305.

47. Bryant, RA, Felmingham KL, Falconer EM, et al. Preliminary evidence of the short allele of the serotonin transporter gene predicting poor response to cognitive behavior therapy in posttraumatic stress disorder. Biol Psychiatry. 2010;67:1217-1219.

48. US Department of Veterans Affairs. National Center for PTSD newsletter. Clinician's Trauma Update CTU online. Available from: http://www.ptsd.va.gov/professional/newsletters/ctu-online.asp. 2010 Oct 10 .

ethical issues; Animal models; Pharmacogenetics; Prenatal diagnosis; Dysmorphology. The manuscript management system is completely online and includes a very quick and fair peer-review system, which is all easy to use. Visit http://www.dovepress.com/testimonials.php to read real quotes from published authors. 